\title{
Cardiac Changes in Hepatic Cirrhosis
}

\author{
${ }^{1}$ Dr. S.Selvamani.MD, ${ }^{2}$ Dr. C.Vignesh, \\ ${ }^{1}$ Assistant Professor, Department Of General Medicine, Coimbatore Medical College \&Hospital. \\ ${ }_{2}^{2}$ postgraduate In General Medicine, Department Of General Medicine, Coimbatore Medical College \&Hospital.
}

\begin{abstract}
Cirrhosis liver. Is characterized by diffuse destruction and renegeration of hepatic parenchymal cells leading to deposition of connective tissue with resulting disorganization of the lobular and vascular architecture. Despite the remarkable regenerative capacity of the liver,once hepatic parenchymal reserve is exceeded,clinically overt or decompensated cirrhosis ensues. Portal hypertension develops when the resistance to blood flow through the liver and resulting increase in portal venous pressure lead to diversion of blood flow through low resistance portosystemic collaterals thereby bypassing the liver.

Hyperdynamic circulatory state is one of the manifestation of portal hypertension. The splanchnic vasodialatation is an important factor that promotes and maintains the portal hypertensive state.This in turn leads to activation of neurohumoral pathways that stimulate renal sodium retention,expansion of plasma volume and ultimately accumulation of ascetic fluid in persons with cirrhosis. Expanded plasma volume also contributrs to increase in portal blood flow and portal pressure. Hyperdynamic circulation more directly suggest the presence of portal hypertension and hepatocellular failure. Studies have been carried out over to evaluate the cardiac and heamodynamic changes in cirrhosis of liver.
\end{abstract}

\section{Introduction}

The current study was designed to precisely evaluate the cardiovascular system in a group of patients with cirrhosis based on clinical examination,electrocardiography roentgrnography and M-Mode 2-dimensional echocardiography

\section{Aim Of The Study}

To clinically evaluate patients with hepatic cirrhosis with respect to changes in heart rate, blood pressure,mean arterial pressure,jugular venous pressure and precordial examination

To document the electrical and morphological alterations in the heart in patients with cirrhosis by means of non-invasive investigations like electrocardiography,roentgenography and M-Mode 2-dimensional echocardiography

To determine the relationship between the cardiac and hemodynamic parameters and the severity and extent of hepatic cirrhosis.

\section{Materials And Methods}

The present study was conducted in the coimbatore medical college hospital,between January 2016 and may 2016.96 patients of cirrhosis liver were selected for the study.these patients were admitted in general medical wards.

Criteria followed for selection of patients included.

\section{Inclusion criteria:}

Only patients with clinical,biochemical and sonographic evidence of cirrhosis liver were selected.

\section{Exclusion criteria:}

patients with previously detected heart disease were excluded from the study

Patients with intercurrent illness on these who were critically ill were excluded from the study.

Patient with cardiac cirrhosis were excluded from the study.

A detailed history was elicited from the patient with special reference to cardiovascular symptoms.A thorough physical examination was performed in the patients and a special note was made regarding heart rate \& rhythm,blood pressure,jugular venous pulse and pressure and precordial examination.

All patients were subjected to routine investigations viz blood urea, sugar,complete haemogram,serum cholesterol\&liver funtion tests.All patients were subjected to ultrasound scan abdomen to conformed the diagnosis of cirrhosis.Liver biospy was performed in 10 patientd who had no condraindications after having obtained a written consent from the patient. Patients with ascites underwent abdominal paracentesis and fluid 
were analysed for protein content and cells.All patients were then subjected to electrocardiography,chest X ray and M-Mode 2 dimensional echocardiography.

\section{Results And Observation}

Out of the 96 patients studied, 48(50\%) were males and 48(50\%) were females. The age of these patients ranged from 24 years to 73 years.

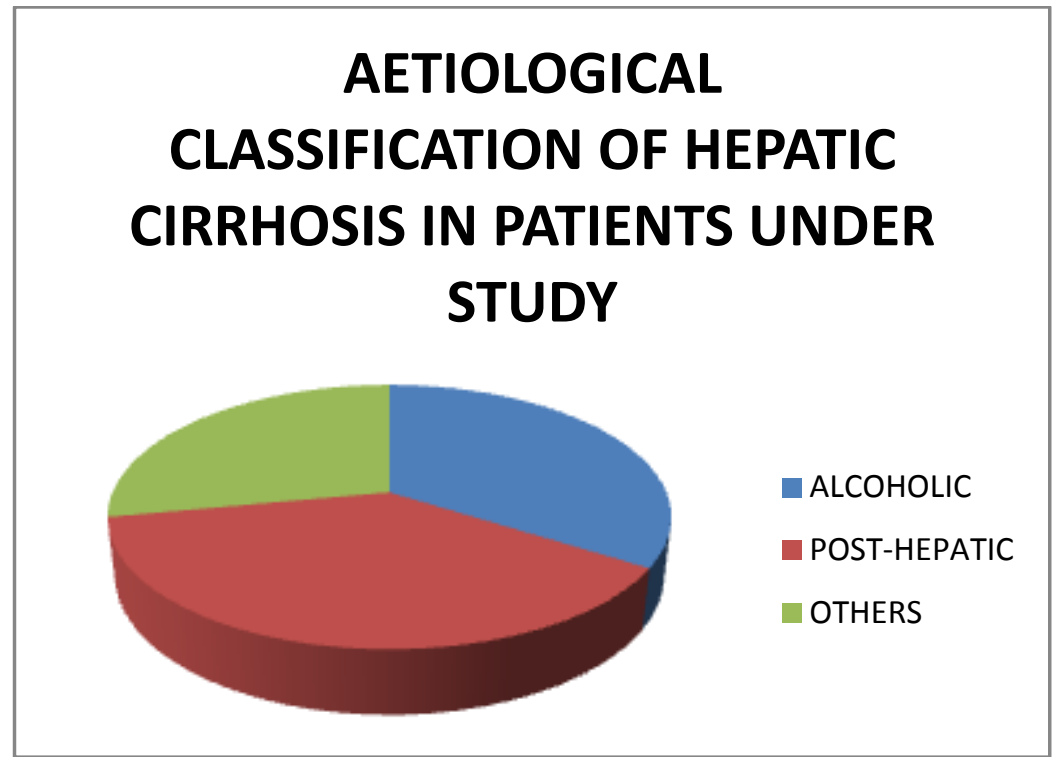

33 patients(34.4\%)were alcoholics, 27 patients(28.1) had past history of jaundice or blood transfusion, 24 patients $(25 \%)$ presented with haemetemesis on admission and 75 patients $(78.1 \%)$ had ascites, 27 patients (28\%)had jaundice and 54 patients $(56.3 \%)$ had clinicaaly detectable splenomegaly on admission. All patients had sonograohic evidence of cirrhosis with portal hypertension and biopsy performed in 30 patients were confirmative of cirrhosis liver.

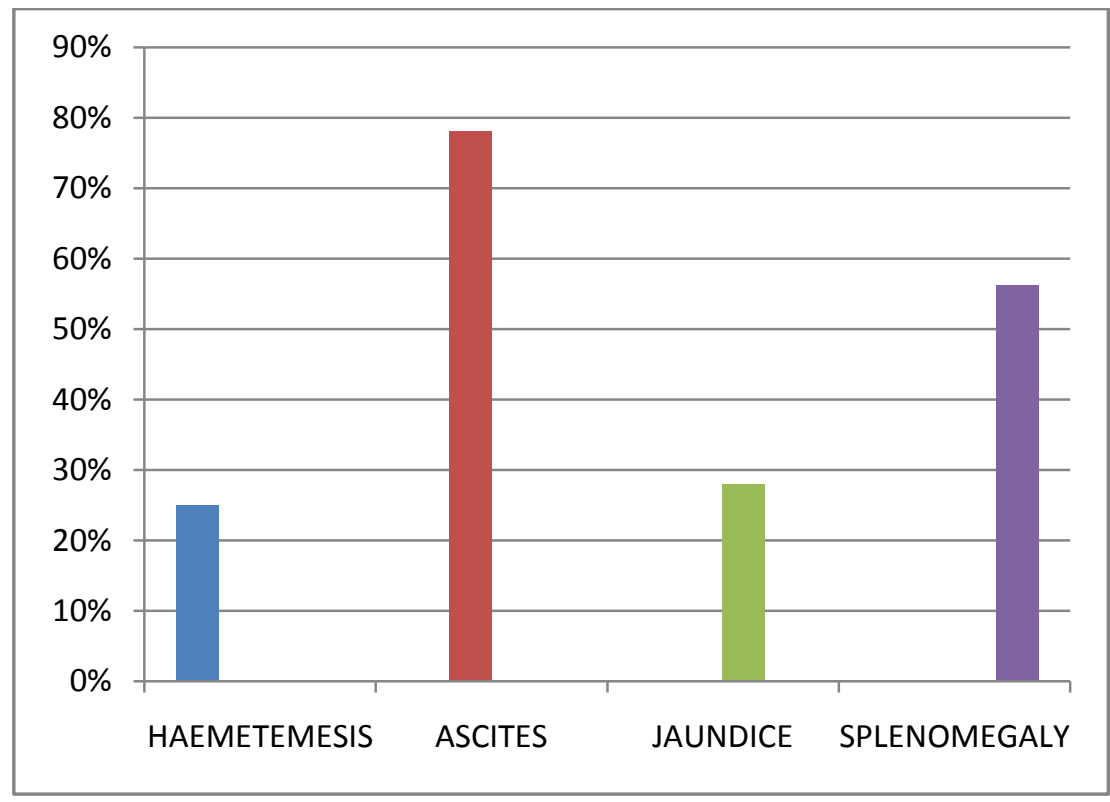

Regarding cardiovascular examination, 42(43.8\%) out of 96 patient had symptoms referable to the heart Of these $24(25 \%)$ were males and 18(18.8\%)were females ,3 patient $(3.13 \%)$ had congestive cardiac failure. The average pulse rate was 87.25 (in males-95.62; and in females 78.87)and it ranged from 54/min to $120 / \mathrm{min}$. The jugular venous pressure was elevated in 21(21.88\%) patients. The systolic blood pressure ranged 
from $90 \mathrm{mmHg}$ to $180 \mathrm{mmHg}$., the average being 119.25 ,(males 125.37 , and females 113.12), The diastolic blood pressure ranged from $50 \mathrm{mmHg}$ to $100 \mathrm{mmHg}$., the average being $71.56(76.88$ in malesand 66.25 in females).The mean arterial pressure ranged from 70 to 110 the average being 84.53 , ( 74.33 for males and 78.33 for females)Functional high output systolic flow murmur was detected in 54(56.3\%) of the patients $24(25 \%)$ males and $30(31.25 \%)$ females.

The electrocardiogram showed average heart rate of 82.83.The QRS axis was in the range of -30 to +60. The low QRS voltage in chest leads and limb leads was found in 6 patients.

Counter clockwise rotation was seen in 15(15.63\%)patients and clockwise rotation was seen in $6(6.25 \%)$ patients. Low voltage QRS complexes were present in $18(18.8 \%)$ patients.T wave inversion was found in chest leads( V1 to V3/V4) in 27 (25\%)patients.Sinus node dysfunction was seen in 3 patients(3.13\%).ventricular premature complexes was seen in 3 patients(3.13\%)and superaventricular premature complex was seen in 3 patients(3.13\%).

\begin{tabular}{|c|c|c|c|}
\hline S.NO & ECHOCARDIOGRAPHIC ABNORMALITIES & INCIDENCE & $\%$ \\
\hline 1 & PERICARDIAL EFFUSION & 81 & 62.5 \\
\hline 2 & CHAMBER ENLARGEMENT & & \\
\hline & $\begin{array}{c}\text { ALL FOUR } \\
\text { LA } \\
\text { RA } \\
\text { RV } \\
\text { LV }\end{array}$ & $\begin{array}{l}6 \\
27 \\
6 \\
3 \\
21\end{array}$ & $\begin{array}{l}6.25 \\
28.12 \\
6.25 \\
3.13 \\
21.88\end{array}$ \\
\hline 3 & $\begin{array}{r}\text { VALVULAR LESIONS } \\
\text { TOTAL } \\
\text { AORTIC } \\
\text { MITRAL } \\
\text { PULMONARY }\end{array}$ & $\begin{array}{l}18 \\
15 \\
3 \\
3\end{array}$ & $\begin{array}{l}18.75 \\
15.63 \\
3.13 \\
3.13\end{array}$ \\
\hline 4 & LV MASS & 6 & 6.25 \\
\hline 5 & LV SYSTOLIC DYSFUNCTION & 3 & 3.13 \\
\hline
\end{tabular}

The chest roentgenogram showed elevated dome of both hemidiaphragms in 72 patients (75\%).cardiomegaly was evident in chest $\mathrm{X}$ ray in 9 patients(9.4\%).

M-Mode 2 dimensional Echocardiographic studies done.,showed a positive abnormality in 81(79.4\%)patients, 12 patients had normal study and echocardiography could not be done in 3 patients who died due to massive haemetemesis.Pericardial effusion was detected in 60(62.5\%)patients, Regarding enlargement of cardiac chambers - all 4 chambers were enlarged in $6(62.5 \%)$ patients,left ventricular enlargement was seen in $21(21.88 \%)$ patients and left ventricular hypertrophy in 6(6.25\%)patients were detected to have co-existing systemic hypertension. Right atrial enlargement was seen in $9(9.38 \%)$ patients. Evidence of left ventricular dysfunction was seen in $3(3.13 \%)$ patients who subsequently succumbed to the disease. Regarding valvular abnormalities, 18 patients $(18.75 \%)$ had coexixting valvular lesions.of these aortic valve lesion was seen in 15(15.63\%)patients(aortic sclerosis in 12 patients;aortic regurgitation in 3 patients and aortic valve thickening in 3 patients).Mitral valve thickening was noted in 3 patients(3.13\%)and pulmonary valve thickening in 3 patients $(3.13 \%)$.

Heart Rate Versus Serum Albumin And Serum Bilirubin

Significant positive correlation was noted between the heart rate and levels of serum bilirubin and significant negative correlation was noted between the heart and serum albumin.

Mean Arterial Pressure Versus Serum Albumin Serum Bilirubin

Significant positive correlation was noted between serum albumin and mean arterial pressure and significant negative correlation between mean arterial pressure and serum bilirubin Mean Arterial Pressure Versus Blood Urea

Significant negative correlation was noted between the mean arterial pressure and blood urea

The average systolic blood pressure, diastolic blood pressure and mean arterial pressure in the present study were 119.25 and $71.56 \mathrm{mmHg}$ and 84.53 respectively.

\section{Conclusion}

1.The results of this study clearly show taht a large number of patients with hepatic cirrhosis though asymptomatic with regard to cardiovascular system, have evidence of cardiac involvement on echo cardiography 2.cardiac decompensation in cirrhosis is rare despite the high output state and its presence as indicated by left ventricular systolic dyfunction is probably a preterminal event. 
3.Ther is a strong association between the severity of the liver disease and the degree of hyperdynamic circulation.

4.Cardiac arrhythmias are rarely encountered and multiple premature ventricular complexes as seen in one patient is probably a preterminal event.

5.Electrocardigraphic abnormalities included left axis due to horizontal heart position,low voltage complexes due to pericardial effusion and non specific $\mathrm{T}$ wave abnormalities.

6.Pericardial effusion was the commonest abnormality detected echocardiogrphically followed by enlargement of cardiac chambers especially left atrium.

7.Valvular abnormalities detected were incidental findings probably not related cirrhosis.

8.There is a probable association between the hyperdynamic circulation in cirrhosis liver and the pathogenesis of hepatorenal syndrome.

9.Cardiac evaluation is a pre-requisite in patients with cirrhosis undergoing stress like surgery because the presence of cardiac involvement adds to the morbidity and mortality....

\section{Bibliography}

[1]. Albillos A;Lled O J L -Haemodynamic effects of alpha-adrenergic blockage with prazosin in cirrhotic patients with portal hypertension-hepatology 1994 sept:20(3)611-7.

[2]. Alvarez D;Golombek D -Diurnal fluctuations of portal and systemic haemodynamic parameters in patients with cirrhosisHepatology 1994 Nov 20(5)1198-203

[3]. Azoulay D et al- ransjugular intrahepatic porto systemic shunts worsen the hyperdynamic circulatory state of the cirrhotic patients;preliminary report of prospective study-Hepatology 1994 Jan;19(1) 129-32.

[4]. Bayer T.D - Cirrhosis of the liver and its major sequelae ch122 pg 786-795 cecil textbook of Medicine.,Edited n=by Wyngarden james B;Smithal.H,BenneH.J.C-19 ${ }^{\text {th }}$ ED 1992

[5]. Bennardi M;Rubboli A; et al - Reduced Cardiovascular responsiveness to exercise-induced sympathoadrenergic stimulation in patients with cirrhosis-Journal of hepatology 1991 Mar 12(2);201-16

[6]. Bernardi M,Trevisani F et al - Chronobiological evaluation of sympatho adrenergic function in cirrhosis - Relationship with arterial pressure and heart rate-Gastroenterology $1987 ; 93 ; 1178$.

[7]. Bihari D - Mismatch of oxygen heply and elemanel in septic shock-Springer Velag Berlin 1988.

[8]. Bihari D, Gimson A.E et al -Lactic acidosis in fulminant hepatic failure -some aspects of pathogenesis \&prognosis-Journal of Hepatology 1(4);405-416;1985.

[9]. Bosch J,Pizeueta Pet al -Pathophysiology of portal Hypertension-Gastroenterology clinics of North America 1992 Mar;21(1) Pg 114.

[10]. Braverman AC;Steiner MA - High output congestive heart failure following TIPS - Chest 1995;may 107(5)

[11]. Robbins Pathologic basis of Disease $-8^{\text {th }}$ ED 2009.

[12]. Dudhley F.J pathophysiology of ascites formation - Gastroenterology clinics of North America -1992 Mar ;21(10);215-236

[13]. Hecker R, Sherlock S - Electrolyte \& circulatory changes in terminal liver failure - LANCET 1956

[14]. Sherlock S -Vasodilatation associated with hepatic cellular disease,relation to functional organ failure-Gut 1990;31;365

[15]. Wernze H Tittor W-Release of prostonoids into the portal \& hepatic -vein in patients with chronic Liver disease - Hepatology 1986-6-911. 